\title{
Monte-Carlo simulations of spinodal ordering and decomposition in compositionally modulated alloys
}

\author{
Michael Atzmon \\ Department of Nuclear Engineering, The University of Michigan, Ann Arbor, Michigan 48109
}

\begin{abstract}
(Received 5 June 1989; accepted 25 August 1989)
The early-stage kinetics of interdiffusion in compositionally modulated films have been studied by Monte-Carlo simulations on an Ising lattice in two and three dimensions, using nearest-neighbor interactions. For a negative heat of mixing and below the order-disorder transition temperature, if a short-wavelength modulation is along a direction that is not consistent with long-range order, then its temporal evolution does not follow the Cahn-Hilliard-Cook theory. The modulation amplitude decreases as a function of time, rather than increases; i.e., no one-dimensional spinodal ordering is observed. This disagreement with the theory implies that ordering in three dimensions cannot be described by a one-dimensional theory.
\end{abstract}

\section{INTRODUCTION}

Ordering kinetics in binary alloys have been the subject of many studies. The first theory was developed by Dienes ${ }^{1}$ and was based on a quasi-chemical approach. More recent studies ${ }^{2-4}$ employ the cluster variation method and assume a vacancy mechanism for atomic diffusion. The history of these theories has been reviewed by Cahn. ${ }^{5}$ Most investigations of diffusion in multilayered materials employ the theory of Cook $e t a l{ }^{6}$ It is a discrete version of the Hillert and Cahn and Hilliard theory, ${ }^{7,8}$ originally developed to describe spinodal decomposition. The results of Ref. 6 have been used extensively to describe the kinetics of ordering in bulk alloys and of interdiffusion in compositionally modulated thin films with short-wavelength modulations. Theories of the migration of anti-phase boundaries $^{9}$ assume local equilibrium and apply therefore only to later stages. In the present study, atomic diffusion at small composition-modulation amplitudes is simulated in a kinetic Ising model. Both ordering and phase-separating alloys are studied and compared to theory and experiment. In contrast to other studies, ${ }^{10,11}$ an atomic interaction potential is assumed rather than a Ginzburg-Landau free energy functional with composition dependence only. This results in a time dependence of the free-energy functional, through its dependence on short-range order. The present study is the first simulation examining the application of the Cahn-Hilliard theory to ordering (negative heat of mixing, $\Delta H$ ). While the theory and simulations are in qualitative agreement for spinodal decomposition, they disagree for ordering alloys below the order-disorder transition temperature. In some crucial cases theory and simulation differ in the sign of the amplification factor. The linear approximation leads to an averaging of the composition over the coordinates perpendicular to the modulation direction. This makes the theory one-dimensional and therefore not applicable to higherdimensional systems.

The temporal evolution of single-phase binary alloys with composition fluctuations have been studied experimentally and theoretically by many investigators. The theoretical treatment is based on a postulate by Hillert $^{7}$ and Cahn and Hilliard, ${ }^{8}$ according to which the free energy functional is expressed as a sum of a composition-dependent bulk term $f_{0}(c)$ and a gradient energy term, proportional to the square of the composition gradient. This approach is based on a continuum assumption; i.e., the wavelength of composition variations is greater than the interatomic spacing. The total free energy is then given by:

$$
F=\int\left[f_{0}(c)+\kappa(\nabla c)^{2}\right] d V
$$

where $\kappa$ is the gradient energy coefficient. The diffusional flux is set proportional to the gradient of the chemical potential, and the resulting diffusion equation can be linearized and solved analytically for smallamplitude composition modulations. If the initial composition is expressed as a Fourier series, then each term evolves independently according to:

$$
\begin{aligned}
A(t) \cdot \cos \boldsymbol{\beta} \cdot \mathbf{r} & \\
& =c_{\beta} \exp \left[-\tilde{D}\left(1+\frac{2 \kappa \beta^{2}}{f_{0}^{\prime \prime}}\right) \beta^{2} t\right] \cos \boldsymbol{\beta} \cdot \mathbf{r}
\end{aligned}
$$

where $\beta=2 \pi / \lambda, f_{0}^{\prime \prime}=\partial^{2} f_{0} / \partial c^{2}, \lambda$ is the modulation wavelength, and $\tilde{D}$ is the bulk interdiffusion coefficient. The amplification factor $R$ is defined as the factor to the left of the time, $t$, in the square brackets. We define $\tilde{D}_{\beta}=R / \beta^{2}$ as an effective, wavelength-dependent interdiffusion coefficient.

The Cahn-Hilliard theory is based on continuum assumptions, and is expected to fail when the composition modulation wavelength is comparable to the inter- 
atomic distance. Cook et al. ${ }^{6}$ extended the theory to include this limit by solving the Cahn-Hilliard equation on a discrete cubic lattice. They obtained the same result as Eq. (2), with $\beta^{2}$ replaced by the dispersion relation

$$
B^{2}=\left(1 / a^{2}\right) \sum_{\text {n. n. }}[1-\cos \boldsymbol{\beta} \cdot \mathbf{r}]
$$

where $a$ is the interplanar spacing, $\mathbf{r}$ is equal to the vector connecting a lattice site to a nearest neighbor, and the sum is over the nearest neighbors. In the continuum limit $(\beta \rightarrow 0), B^{2}$ approaches $\beta^{2}$. For wave vectors along $(100)$ or $(110)$ in bcc or along (100) or (111) in fcc, Eq. (3) is reduced to $B^{2}=\left(2 / d^{2}\right)[1-\cos (2 \pi d / \lambda)]$, where $d$ is the interplanar spacing.

As a result of the competition between bulk and gradient energy, the amplification factor $R$ undergoes a sign change at a critical value of the modulation wavelength, $\lambda_{c}$. The corresponding critical value of $B_{c}^{2}$ is obtained by setting the amplification factor equal to zero, yielding $B_{c}^{2}=-f_{0}^{\prime \prime} / 2 \kappa$. For a positive heat of mixing, $R>0$ below a critical wavelength, $\lambda_{C}$, and $R<0$ above it; i.e., spinodal decomposition takes place. Any composition fluctuation with $\lambda>\lambda_{C}$ is unstable and grows. For a negative heat of mixing, $R>0$ for $\lambda<\lambda_{C}$ and $R<0$ for $\lambda>\lambda_{c}$. In the latter case, the apparent phase separation at short wavelengths has been explained by Cook et al. ${ }^{6}$ as spinodal ordering, where the long-range order is described as a short-wavelength composition modulation.

The theory described above has been used extensively in the analysis of the early stages of spinodal decomposition, as well as for interdiffusion in compositionally modulated thin films. ${ }^{12,13}$ In the latter case, the initial state is that of a small-amplitude modulation in one dimension, and its temporal evolution can be studied by diffraction methods. In Ref. 6 , the authors apply their discrete theory to describe the kinetics of the initial stages of ordering. The theory is described as three dimensional, but strictly speaking, it is one dimensional: as a result of linearization, each atom is assumed to diffuse in the potential resulting from an atomic composition averaged over lattice planes perpendicular to the modulation direction. However, in an ordered alloy, the planes perpendicular to a particular modulation direction may all have the same average composition. Hence, the amplitude of a planewave modulation does not necessarily give a complete description of long-range order. As an example, consider ordered lattices of $\mathrm{Cu}_{3} \mathrm{Au}$ and $\mathrm{CuAu} .{ }^{14}$ The former consists of alternate (001) planes which contain only $\mathrm{Cu}$ or only $\mathrm{Au}$ atoms. This ordered structure is fully described by a (001) plane-wave modulation. The ordered $\mathrm{Cu}_{3} \mathrm{Au}$ lattice consists of $\mathrm{Cu}$ atoms on the face centers and $\mathrm{Au}$ atoms on the corners of the unit cell. $\mathrm{A}$ (001) plane wave modulation description of this order is incomplete because it does not contain information on the atomic arrangements within the planes (every other plane contains $50 \% \mathrm{Cu}$ and $50 \% \mathrm{Au}$ and is ordered). In the (111) direction, the modulation amplitude is zero because all planes have the same average composition. These observations suggest that a one-dimensional theory is not adequate for describing the ordering process, which is three dimensional. In order to study this question, we have performed a Monte-Carlo simulation of interdiffusion on a discrete lattice, and compared the results to theory and experiment. A number of authors have used Monte-Carlo simulations to study various other aspects of diffusion in solids, as reviewed in Ref. 15. Our results show that for a negative heat of mixing, below the order-disorder transition temperature, and for a modulation in a crystallographic direction which is not consistent with long-range order, modulations of all wavelengths decay in time. In contrast to this absence of one-dimensional spinodal ordering, the simulation of a system with positive heat of mixing is qualitatively consistent with the theory. In addition, the simulations show that the development of short-range order during the interdiffusion process gives rise to deviations from regular solution behavior, and therefore to nonlinearity.

\section{MONTE-CARLO SIMULATIONS}

Simulations were carried out in a binary Ising model with conserved order parameter ${ }^{15}$ for square, triangular (2 dimensions), fcc, or bcc (3 dimensions) structure. Each lattice site was occupied by a type A or B atom, with a conserved total composition. It should be stressed that this model is different from that of a ferromagnet or antiferromagnet (nonconserved order parameter ${ }^{15}$ ), in which the total number of "up" (or "down") sites is not conserved. The latter is appropriate for describing domain-wall migration in an ordering alloy after local equilibrium is established, a regime studied by Allen and Cahn. ${ }^{9}$ The arrays had $1.6 \cdot 10^{5}$ sites in two dimensions and $1 \cdot 10^{5}-9 \cdot 10^{5}$ in three dimensions. A plane-wave, sinusoidal initial composition modulation was imposed on each sample, with modulation wavelengths, $\lambda$, of 2 to 20 monatomic layers and amplitudes of $0-0.5$. The size of the sample in the direction of the modulation was always selected to be an integer multiple of $\lambda$. The atomic arrangement within each layer perpendicular to the modulation direction was random, and an average sample composition $c$ of 0.160.5 was selected. The energy of the lattice was calculated by assigning values to nearest-neighbor (n.n.) bonds as follows: for a negative heat of mixing, $\Delta H,-1$ for unlike n.n. and +1 for line n.n.; for a positive heat of mixing, the opposite signs. Atomic diffusion was simulated by selecting a random atom and a random n.n., and exchanging them with the probability $\exp (-\Delta E / k T) /\{1+\exp (-\Delta E / k T)\}$, where $\Delta E$ is the 
energy change due to an atomic exchange and $k T$ the temperature in energy units. Periodic boundary conditions were applied. The output of the random number generator was scrambled following Ref. 16 in order to ensure a practically random sequence. $k T$ values of 0.2 and 0.5 were studied, well below the order-disorder transition temperature ( 8 for bcc and 4 for fcc, according to the Bragg-Williams theory ${ }^{17}$ ). The evolution of the amplitude as a function of the number of MonteCarlo steps was followed by calculating the structure factor $S(\mathbf{q})=2 / L \int_{0}^{L}\left[c(x)-c_{0}\right] \sin (q x) d x$, where $q=$ $2 \pi / \lambda, \mathbf{q}$ is parallel to the modulation direction, and $L$ is the sample size in the modulation direction. The effective interdiffusion coefficient was calculated according to Eq. (2) and averaged over 25 runs, each consisting of approximately 0.2 Monte-Carlo steps per site. It is presented in units of $a^{2} \Gamma$, where $a$ is the interatomic spacing and $\Gamma$ is the jump frequency in all directions. The error was determined from the scatter in the individual results. The short-range order (SRO) parameter was defined as the average number of line n.n. bonds minus the number of unlike n.n. bonds per atom, which is equal to the average energy per atom. Milchev et al. ${ }^{18}$ have shown that for sampling thermal fluctuations in a nonequilibrium system, the error does not decrease with increasing sample size. The present simulations do not show such lack of self-averaging, because the initial, preseeded fluctuation in this study is well defined.

\section{RESULTS AND DISCUSSION}

In two dimensions, both the triangular and the cubic structure showed a nonlinear decay of the logarithm of the amplitude $\log (A)$ as a function of time, the latter being shown in Fig. 1. After the rate of change of the SRO parameter becomes negligible, $\log (A)$ can be well approximated with a linear function. The nonlinear behavior was observed to be more pronounced for larger initial amplitudes. This can be explained on the basis of kinetic slowdown of the diffusion kinetics with increasing SRO (see Fultz ${ }^{19}$ ): as SRO develops, atomic jumps become increasingly more difficult because the atoms are trapped in deeper potential wells. In the present study, the smaller the initial amplitude $A$, the sooner does each volume element reach the average composition and the final $\mathrm{SRO}$, and therefore the slower the decay rate of $\operatorname{In}(A)$. There is also a thermodynamic contribution to the composition dependence of the interdiffusion coefficient, a discussion of which will be published elsewhere. The deviation of $\operatorname{In}(A)$ vs $t$ from linearity is not a result of noise, ${ }^{20}$ because it is more pronounced at large amplitude, whereas the effect of noise is dominant at small modulation amplitudes. Marro et al. ${ }^{21}$ have observed a nonlinearity in a similar simulation of spinodal decomposition, and we attribute it to the same effect of SRO.

In three dimensions, one also observed nonlinear decay of $\log (A)$, but in this case no linear regime is observed. Because of the slower atomic jump kinetics in ordered regions, diffusion occurs primarily along antiphase boundaries (APB). Therefore, the timedependence of the interdiffusion coefficient depends directly on the kinetics of APB migration, which will be discussed in a future publication. The effective interdiffusion coefficient was determined from the initial

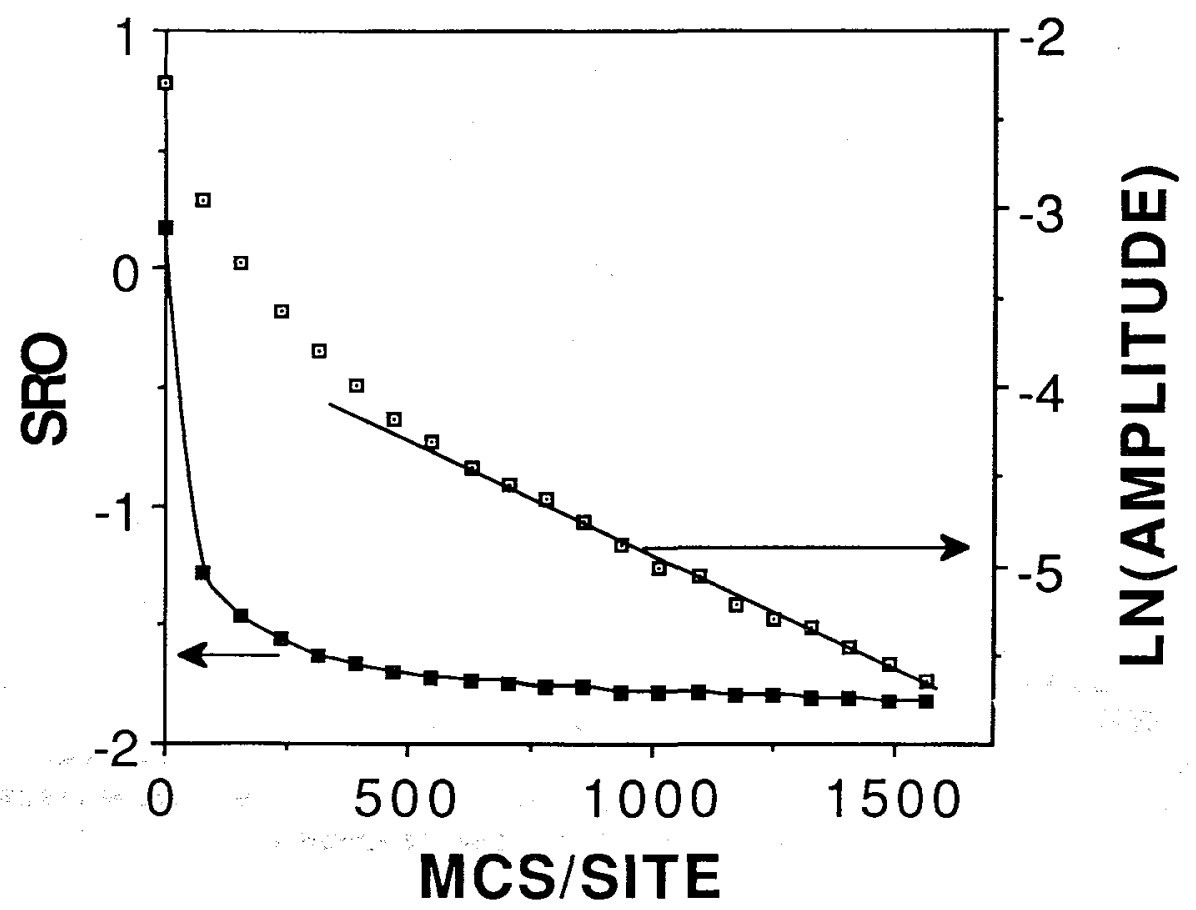

FIG. 1. The logarithm of the modulation amplitude as a function of the normalized time (Monte-Carlo steps per site) for a two-dimensional, square lattice, modulated in the (11) direction. The modulation wavelength is 40 atomic rows, the temperature, 1.5 , equal to $3 / 8$ times the order-disorder transition temperature, and the average composition is equiatomic. The short-range order parameter is also plotted. The initial amplitude is 0.2 . The error is smaller than the points marked. 
slope (0-0.2 Monte-Carlo steps per site) of $\log (A)$ and initial amplitude of $A=0.05$. During this short time, the short-range order did not change significantly.

In Fig. 2, the effective interdiffusion coefficient $\tilde{D}_{B}$, at $k T=0.2$, is plotted against $B^{2}$ for a negative and positive heat of mixing, $\Delta H$, of the same magnitude. The structures shown are (111)-modulated fcc and (100)and (110)-modulated bcc. For the fcc structure with (111) modulation and $\Delta H>0$, spinodal decomposition $\left(\tilde{D}_{B}<0\right)$ is observed above a critical wavelength, in qualitative agreement with the theory. For $\Delta H<0$ in the same structure, $\tilde{D}_{B}$ is positive, i.e., existing modulations decay, for all possible wavelengths. Similar behavior is observed for bcc with (110) modulation. For $\Delta H<0$ in the bcc structure, $\tilde{D}_{B}$ is negative for the shortest wavelength and positive otherwise. In two dimensions (not shown), for $\Delta H<0, \tilde{D}_{B}$ is always positive for a triangular lattice and for a square lattice with a (10) modulation. It is negative, however, for the shortest wavelength in a (11)-modulated square lattice.

A comparison with estimates ${ }^{12}$ of $\kappa$ and $f_{0}^{\prime \prime}$ will now be used to show that the theory fails to predict the correct critical wavelength. In the low-temperature limit, the entropic driving force for diffusion is negligible and the enthalpy dominates $f_{0}^{\prime \prime}$. Since then both $f_{0}^{\prime \prime}$ and the interfacial energy coefficient, $\kappa$, scale with the heat of mixing parameter $\Omega$ [where $\Delta H=\Omega c(1-c), c$ being the composition], the same critical wavelength is expected for a positive and negative heat of mixing of the same magnitude [see Eq. (3)]. Following Ref. 12, a critical value of $d^{2} B^{2}=4$ is obtained for both fcc and bcc. For the fcc lattice and $\Delta H>0$, the simulation gives $d^{2} B_{c}^{2}=3.64$, in moderate agreement with the theory. For $\Delta H<0$, however, $\tilde{D}_{B}>0$ for all allowed values of $B$, and no critical wavelength is observed. It is seen that for $\Delta H>0$, the results agree qualitatively with the prediction of Cook et al., ${ }^{6}$ but for $\Delta H<0$, the theory does not predict the correct sign of the amplification factor. It is concluded that a theory of ordering in systems with a dimension higher than one will have to take into account the higher-dimensional nature of the process. For $\Delta H<0$ in a (100)-modulated bcc lattice, $\tilde{D}_{B}$ is negative for the shortest possible wavelength (=two monatomic layers), but the critical wavelength still differs drastically from that of the same system with $\Delta H>0: d^{2} B^{2}=3.40$ for $\Delta H>0$ and 10.30 for $\Delta H<0$. In this case, the negative effective interdiffusion coefficient, i.e., the growth of an existing onedimensional fluctuation, is possible for a wavelength equal to two monatomic layers because it is consistent with the increase in long-range order in the alloy. Longrange order is not consistent with a (110) modulation in

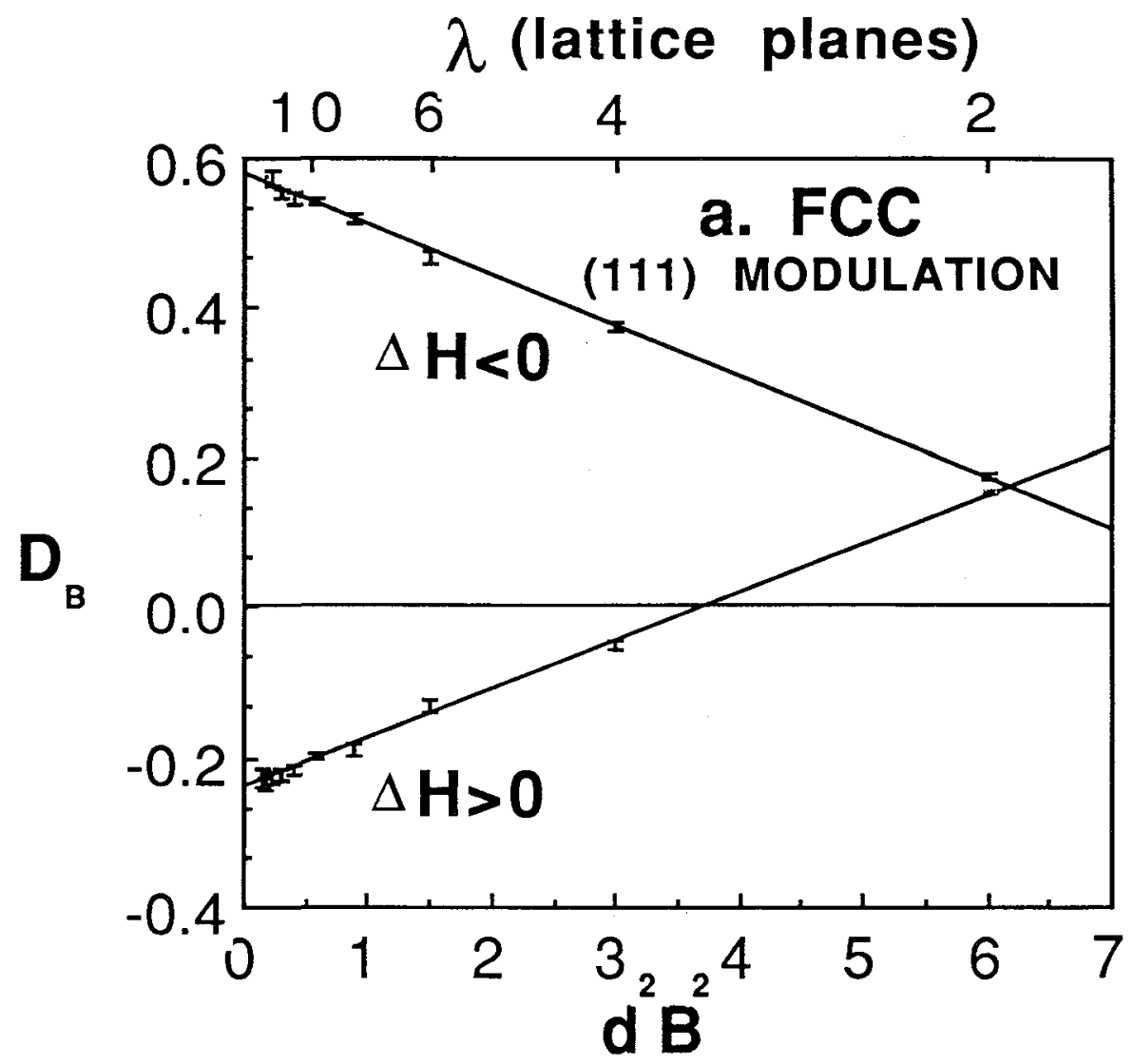

FIG. 2. The effective interdiffusion coefficient $\tilde{D}_{B}$ as a function of $d^{2} B^{2}$ for a three-dimensional lattice with an equiatomic average composition, for a positive and negative heat of mixing. The temperature is 0.2 , equal to 0.025 times the order-disorder transition temperature $T_{c}$ for bcc and 0.05 times $T_{c}$ for fcc. (a) Facecentered cubic with a (111) composition modulation. Note the similarity in the values of $\tilde{D}_{B}$ for a positive and negative $\Delta H$ at $\lambda=2$ lattice planes. (b) Body-centered cubic with a (110) modulation. (c) Bodycentered cubic with a (100) modulation. $d^{2} B^{2}=[6$ in (a) and (b) and 12 in (c) $]$ corresponds to the shortest possible modulation wavelength (two atomic planes).

(a) 

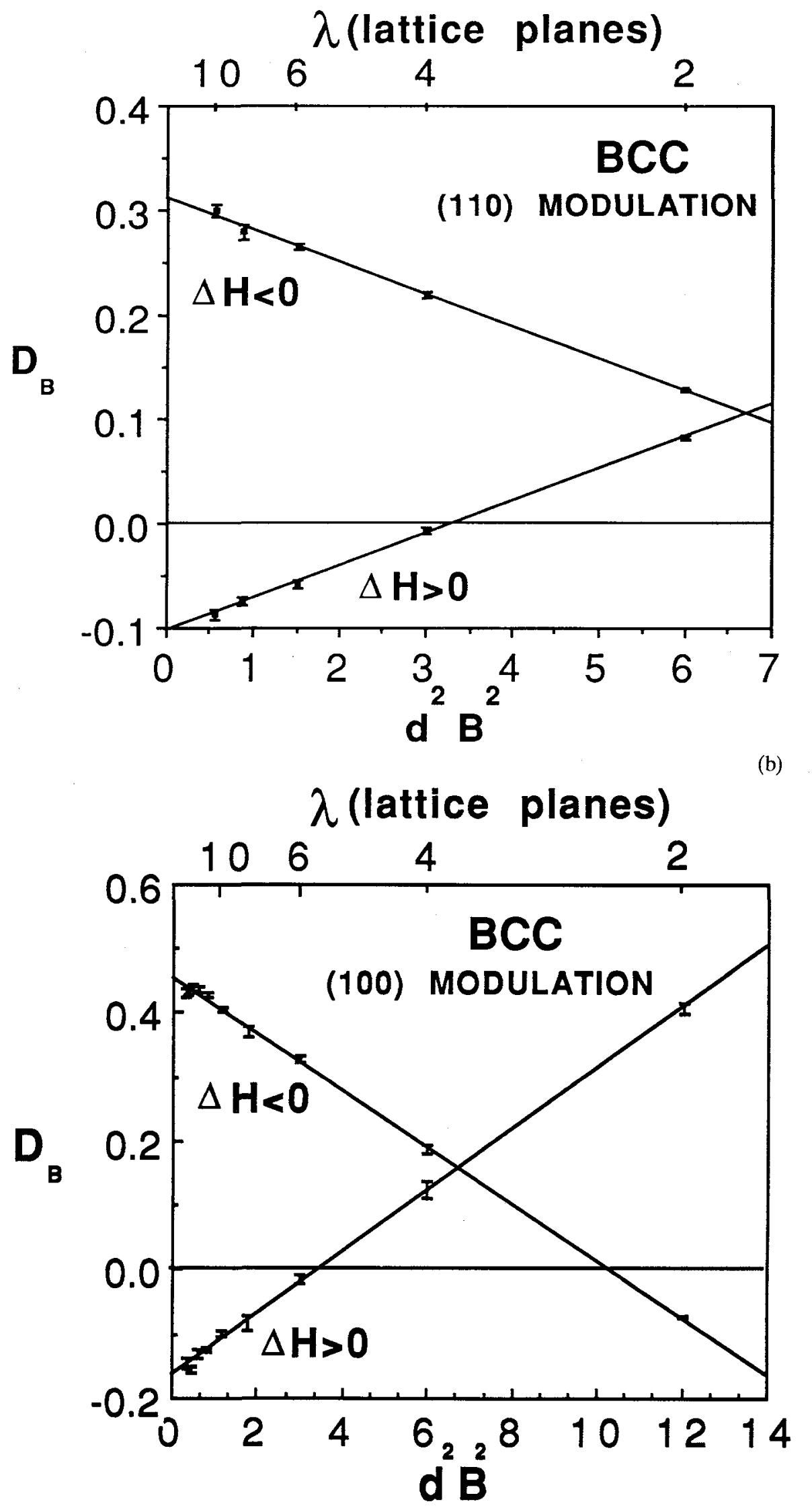

(b)

FIG. 2. (continued) 
a bcc lattice, and indeed, when $\Delta H<0, \tilde{D}_{B}$ is positive for all modulation wavelengths in this structure. Similar reasoning applies to the difference between (10) and (11) modulations in a square lattice.

As mentioned earlier, the one-dimensionality of the Cook et al. ${ }^{6}$ theory is a result of the mean-field and linear approximations. The authors use a linear response approximation, setting the diffusional flux between nearest neighbor sites as proportional to the chemical potential difference between them [Ref. 6 , Eq. (6)]. The use of composition-dependent free-energy functional constitutes a mean-field approximation and, by assuming linear response, the authors implicitly assume small composition fluctuations. The combination of the two approximations leads to the one-dimensionality of their model. Because of the discreteness of the lattice, the composition difference between adjacent sites is 0 or 1 , and the assumption of small composition variations breaks down. The limited validity of the linear response approximation will be discussed in detail in a future publication. ${ }^{22}$

It is interesting to note that for the fcc lattice, $\tilde{D}_{B}$ for the shortest wavelength is the same for $\Delta H>0$ and $\Delta H<0$, and equal to the bulk diffusion coefficient at high temperatures $(1 / 6)$. This is a result of the fact that for the (111) modulation, an atom's number of nearest neighbors at neighboring planes is equal to their number in its own plane. When the modulation wavelength is equal to two interplanar spacings, the total bond energy does not change (in a mean-field sense) when atoms in different planes exchange positions, and the net chemical contribution to the driving force is zero. The same reasoning should apply to (110) modulated bcc [Fig. 2(b)], but we suggest that because of the smaller number of nearest neighbors, the mean-field approximation is less exact and therefore the values of $\tilde{D}_{B}$ differ for different signs of $\Delta H$.

Experimental results are consistent with the present simulations. Measurements of compositionally modulated films are of $\mathrm{Cu}_{84} \mathrm{Au}_{16}{ }^{23}$ and amorphous $\mathrm{Ni}_{55} \mathrm{Zr}_{45}{ }^{24}$ show no negative effective interdiffusion coefficient at short modulation wavelengths, although expected from extrapolation from longer wavelengths. The present work does not reproduce, however, the experimentally observed deviation from linearity in the $\tilde{D}_{B}$ vs $B^{2}$ plots. It is possible that interactions beyond the nearest neighbors will account for this nonlinearity.

\section{CONCLUSION}

We have used Monte-Carlo simulations on an Ising lattice to study interdiffusion in compositionally modulated films of both segregating and ordering alloys. A plane-wave modulation has been preseeded and its temporal evolution investigated. The use of atomic interactions, rather than the assumption of a particular func- tional form of the free-energy functional, allows the identification of the role of ordering in the interdiffusion process. For phase-separating alloys, the results are in qualitative agreement with existing theories, but for ordering alloys below the order-disorder transition temperature, the predicted one-dimensional spinodal ordering is not observed when inconsistent with long-range order. This implies that a truly three-dimensional theory is necessary to describe ordering.

\section{ACKNOWLEDGMENTS}

The author would like to thank F. Spaepen and D. Srolovitz for useful discussions, and the referees for valuable comments. The work has been supported by the Office of Naval Research under contract no. N00014-85-1-C-0023 and by an IBM post-doctoral fellowship in the initial stages, and by funds of The University of Michigan.

\section{REFERENCES}

${ }^{1}$ G. J. Dienes, Acta Metall. 3, 549 (1955).

${ }^{2}$ H. Sato, Acta Metall. 24, 797 (1976).

${ }^{3}$ K. Gschwend, J. Chem. Phys. 69, 5006 (1978).

${ }^{4}$ J. K. McCoy, Phys. Rev. B25, 1734 (1981).

${ }^{5}$ R.W. Cahn, in Phase Transitions in Condensed Systems Experiments and Theory, edited by G. S. Cargill, F. Spaepen, and K-N. Tu (MRS, Pittsburgh, PA, 1987), p. 385.

${ }^{6}$ H. E. Cook, D. de Fontaine, and J. E. Hilliard, Acta Metall. 17, 765 (1969).

${ }^{7}$ M. Hillert, Sc.D. Thesis, Massachusetts Institute of Technology, Cambridge, MA, 1956.

${ }^{8}$ J.W. Cahn and J. E. Hilliard, J. Chem. Phys. 28, 258 (1958).

${ }^{9}$ S. M. Allen and J.W. Cahn, Acta Metall. 27, 1085 (1979).

${ }^{10}$ K. R. Elder, T. M. Rogers, and R. C. Desai, Phys. Rev. B38, 4725 (1988).

${ }^{11}$ A. Milchev, D.W. Heermann, and K. Binder, Acta Metall. 36, 377 (1988).

${ }^{12}$ A. L. Greer and F. Spaepen, in Synthetic Modulated Structures, edited by L. L. Chang and B.C. Giessen (Academic Press, 1985), p. 419.

${ }^{13}$ T. Tsakalakos, Thin Solid Films 86, 79 (1981).

${ }^{14}$ C. S. Barrett and T. B. Massalski, Structure of Metals (McGrawHill, New York, 1966), pp. 272-273.

${ }^{15} \mathrm{~K}$.W. Kehr and K. Binder, in Applications of the Monte Carlo Method in Statistical Physics, edited by K. Binder (Topics in Applied Physics, Springer-Verlag, Berlin, 1984), p. 198.

${ }^{16}$ W.T. Press, W.T. Vetterling, S. Teukolsky, and B. P. Flannery, Numerical Recipes (Cambridge University Press, New York).

${ }^{17}$ R. A. Swalin, Thermodynamics of Solids (Wiley, New York, 1972), pp. $153-159$.

${ }^{18}$ A. Milchev, K. Binder, and D.W. Heermann, Z. Phys.-Condensed Matter 63, 521 (1986).

${ }^{19}$ B. Fultz, J. Chem. Phys. 87, 1604 (1987).

${ }^{20}$ H. E. Cook, Acta Metall. 18, 297 (1970).

${ }^{21}$ J. Marro, A. B. Bortz, M. H. Kalos, and J. L. Lebowitz, Phys. Rev. B12, 2000 (1975).

${ }^{22} \mathrm{M}$. Atzmon (unpublished research).

${ }^{23}$ W. M. Paulson and J. E. Hilliard, J. Appl. Phys. 48, 2117 (1977).

${ }^{24}$ M. Atzmon and F. Spaepen, in Multilayers: Synthesis, Properties and Non-Electronic Applications, edited by T.W. Barbee, F. Spaepen, and L. Greer (MRS, Pittsburgh, PA, 1988), p. 277. 